\title{
IMPLEMENTATION OF PI, FUZZY \& ANN CONTROLLERS TO IMPROVE DYNAMIC RESPONSE OF VECTOR CONTROLLED INDUCTION MOTOR DRIVE
}

\author{
M.Ankarao ${ }^{1}$, M.Vijaya Kumar ${ }^{2}$ \\ ${ }^{I}$ Assistant Professor, Department of EEE, JNTUACE Ananthapur, Andhra Pradesh, India \\ ${ }^{2}$ Professor, Department of EEE, JNTUACE Ananthapur, Andhra Pradesh, India
}

\begin{abstract}
Nowadays, vector controlled induction motor drives with variable speed applications are widely used in order to achieve good dynamic performance and wide speed control. In this paper a new method of controlling technique based on Artificial Neural Network is proposed to improve the speed control of indirect vector controlled induction motor drive. Indirect vector controlled induction motor with conventional PI controller is developed and is replaced with Fuzzy logic controller to overcome the problem of overshoot occurred in conventional PI controller. To obtain quick steady state response and better speed control, ANN technique is proposed and implemented using MATLAB/Simulink. In this paper the speed, torque and stator voltage responses with conventional PI controller, Fuzzy logic controller and proposed artificial neural network based controller are compared and found that the proposed ANN based controller showed increased dynamic performance.
\end{abstract}

Keywords: ANN, FLC, PI controller, IVCIM

\section{INTRODUCTION}

Induction motor is well known as the workhorse of the industry because of its enormous advantages. There was various speed controlling techniques of induction motor drives that are developed in order have better dynamic performance. The growth of the variable speed induction motor drives has a long history more than four decades. From past decades, induction motors are widely used in industrial applications and its speed is controlled using Scalar control technique. In scalar control, the name itself indicates that the magnitude of control variables can only be varied to control the speed of the drive. Flux can be controlled by varying machine voltage and the torque can be controlled by Frequency or slip variations. Use of scalar control limits the performance of the machine because of inherent coupling effect which gives sluggish response and the system is prone to instability. With the fastest developments in power electronics, microprocessors and digital electronics, electric drives are now became more reliable, compact and efficient. In contrast to scalar control, the advancements in electric drive systems made a new invention of vector control technique by Prof. Blashke in early 1972. Scalar control importance has diminished due to the superior performance obtained by Vector control. Out of different methods of speed control of induction motor such as variable frequency, pole changing, variable rotor resistance and stator voltage, constant v/f control, slip recovery methods, the most commonly used method is closed loop constant v/f control.

Just like in Dc motor drives, Vector control of AC drives allows the independent control of torque and flux components. In vector control scheme, the stator phaser currents are resolved into two components and individual torque and flux control is possible. The vector control schemes are classified based on the way of calculating field angle. The Direct vector control method is that in which the field angle is obtained from the terminal voltages and currents and the method where field angle is obtained using rotor position measurement is known as indirect vector control. In this paper, the indirect vector control of induction motor is implemented using the system equations that are assembled as the speed controller, vector controller, inverter, switching logic and the machine with currents, speed and position feedbacks. In conventional method, a PI controller is used for processing the speed error between reference speed and speed feedback signals. PI controller is replaced with Fuzzy Logic Controller and the responses are compared. ANN got lot of demand over the last few years as a powerful technique to solve many real world problems. Neural networks and fuzzy logic techniques are quite different with unique capabilities such as mapping with degree of imprecision, information processing, control of non linear system to a degree that is not possible with conventional control systems.

There is extremely large improvement in techniques used for the controlling of speed of induction motor and for the improved performance of the induction motor drive. The present paper produces an improved ANN based controlling technique which is better than the FLC technique is implemented using MATLAB/Simulink. The various function blocks and equations for the modeling of ANN and FLC based speed controlling for the drive is developed in MATLAB and the results are analyzed. 


\section{INDUCTION MOTOR DRIVE}

Generally, AC drive systems are nonlinear, multivariable and have internal coupling effect and so its stability analysis is very complex. The foregoing problem can be eliminated by using computer simulation, which is very useful in finding the performance of the drive. A simple speed controlling block diagram of an induction motor drive is shown in fig 1.

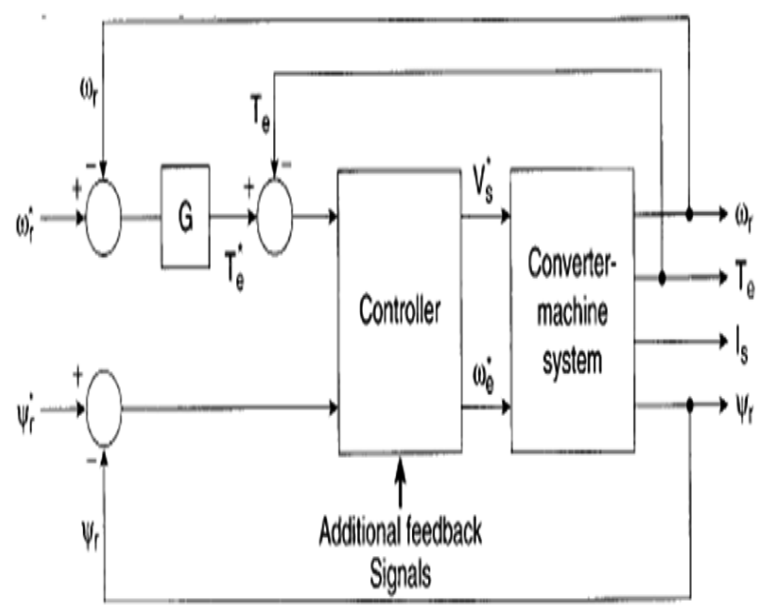

Fig 1 General speed control block diagram

In the above fig 1, the inner torque loop is optional. Inner loop with high gain can provides ability to control the signals in safe limits.

\subsection{Modeling}

Induction motor simulink modeling is developed using two phase motor in direct axis and quadrature axis. With the help of voltage and flux linkages relations in the selected reference frame, the state space model of induction motor is developed in stationary reference frame in this paper. The required equations for modelling are as below.

$$
\begin{gathered}
\frac{d i_{d s}}{d t}=-\gamma i_{d s}+\beta \omega_{y} \lambda_{q r}+\beta \alpha \lambda_{d r}+\beta_{1} V_{d s} \\
\frac{d i_{q s}}{d t}=-\gamma i_{q s}-\beta \omega_{r} \lambda_{q r}+\beta \alpha \lambda_{q r}+\beta_{1} V_{d s} \\
\frac{d \lambda_{d r}}{d t}=-\alpha \lambda_{d r}-\omega_{r} \lambda_{q r}+\alpha L_{m} i_{d s} \\
\frac{d \lambda_{q r}}{d t}=-\alpha \lambda_{q r}+\omega_{r} \lambda_{d r}+\alpha L_{m} i_{q s}
\end{gathered}
$$

Where $\gamma=\left[\frac{{L_{M}^{2}}^{2} \gamma_{Y}+L_{F}^{2} \gamma_{s}}{\sigma L_{s} L_{F}^{2}}\right]$

$\sigma=1-\left[\frac{L_{M}^{2}}{L_{s} L_{W}}\right]$,

$\beta=\frac{L_{M}}{\sigma L_{S} L_{r}}, \quad \beta_{1}=\left[\frac{1}{\sigma L_{s}}\right], \alpha=\left[\frac{V_{Y}}{L_{r}}\right]$
And the torque developed is

$$
\begin{aligned}
& T_{e}=\left(\frac{3}{2}\right)\left(\frac{p}{2}\right) \frac{L_{m}}{T_{T}}\left(i_{q s} \lambda_{d r}-i_{d s} \lambda_{q r}\right) \\
& T_{e}=\left(\frac{3}{2}\right)\left(\frac{p}{2}\right) \frac{L_{m}}{L_{r}} \varphi_{r} i_{q s}
\end{aligned}
$$

Any fluctuations rotor flux linkages results in oscillations in Torque which leads to oscillations in speed.

\subsection{Reference Frames}

The transformations that are performed on voltages and currents to transfer from two phase to three phase or vice versa are Reference frames. Stationary, rotating and synchronous reference frames are generally used for transformation. The transformation that involves converting stator variables into a synchronously rotating reference frame fixed in the rotor is called Park's transformation.

$$
\left[\begin{array}{l}
i_{d s} \\
i_{q s}
\end{array}\right]=\left[\begin{array}{cc}
\cos \theta_{e} & -\sin \theta_{e} \\
\sin \theta_{e} & \cos \theta_{e}
\end{array}\right]\left[\begin{array}{l}
i_{\alpha} \\
i_{\beta}
\end{array}\right]
$$

H.C Stanley showed that time varying Inductances in the voltage equations of an induction motor due to electric circuits in relative motion can be eliminated by transforming the rotor variables to a stationary reference frame fixed on the stator. Later, G. Kron proposed a transformation of both stator and rotor variables to a synchronously rotating reference that moves with the rotating magnetic field.

\section{IMPLEMENTATION OF INDIRECT VECTOR CONTROL}

The speed of the induction motor can be controlled using direct vector control and indirect vector control. Indirect vector control is almost similar to direct vector control except that the field angle is measured using rotor position and the unit vector signals are generated in feed forward manner. Here, generally vector controller accepts the torque and flux commands and then generates components of stator currents and slip angle $\theta_{\text {sl. }}$ Required field angle is obtained by adding the slip angle and rotor angle.

$$
\begin{aligned}
& \theta_{\mathrm{e}}=\theta_{\mathrm{r}}+\theta_{\mathrm{sl}}=\int \mathrm{W}_{\mathrm{e}} \mathrm{dt} \\
& \theta_{\mathrm{sl}}=\int_{\mathrm{W}_{\mathrm{sl}}} \mathrm{dt} \\
& \omega_{s l}=\frac{L_{m} R_{r}}{\varphi_{r} r} i_{q s}
\end{aligned}
$$

Then the $\mathrm{d}-\mathrm{q}$ axis currents are obtained from the torque and flux producing components of stator current as in Eq. 7. The principle of indirect vector control can be understood with the help of Fig 2. 


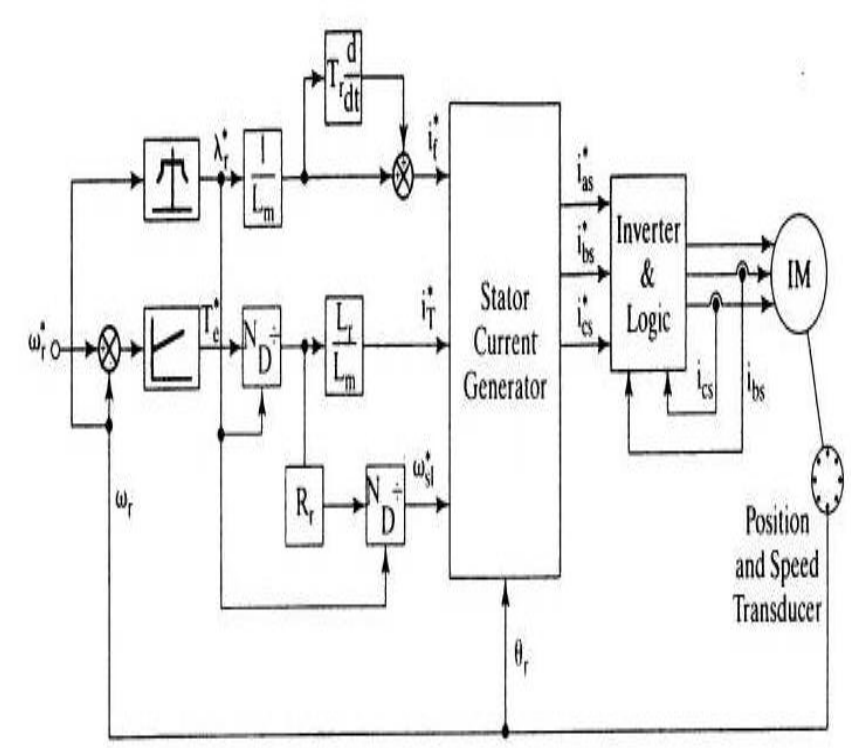

Fig 2. Indirect vector control

In this control, the flux is maintained at rated value upto rated speed. Above that, the flux is weakened in order to keep power output as constant. Vector controller is tuned using exact values of rotor resistance, mutual inductance and rotor self inductance. Tuning of the controller is simple if the motor parameters are constant but these parameters undergo changes.

To implement indirect vector control and to estimate rotor flux, the above mentioned equations $8,9,10$ are used. The outer speed loop generates torque producing component and similarly the flux producing component is obtained. Speed control range in induction motor drives can be extended from standstill to field-weakening region. in this case flux control is must. The flux is maintained constant in constant torque region and in field-weakening region it is programmed such that inverter always operates in PWM mode.

Hysteresis band PWM is used for current control in which actual current continuously follows the command current within a hysteresis limit. The output of this is fed as signals to the SVM technique and the required modulated voltage signals are obtained. The resulted pulses are then converted according to the transformations required. SVM technique is little complex and computation intensive and so the available PWM switching frequency is limited. To overcome this ANN based SVM technique can be used in further future.

\section{CONTROLLERS DESIGN}

The $\mathrm{Kp}$ and $\mathrm{Ki}$ gain values of the controller are tuned by using the common technique of Zeigler-Nicholas tuning method is used. In case of PI controller, if the gains exceeds certain value then the command torque variations increases and destabilizes the system. Because of continuous variation of machine parameters, fixed gain of PI Controllers varies. And it is Unable to provide desired control performance.
Speed controller is necessary to control the speed of the induction motor drive. Design of this speed controller greatly affects the performance of the electric drive. PI controllers are the most commonly used speed controllers before the introduction of fuzzy controller. Design and tuning of the controllers are defined in this section.

\subsection{Propotional-Plus-Integral Controller}

Controllers are used to keep the motor speed at required setpoint. PI controller has a property of Zero steady state error as the type 1 transfer function is used. The actual speed and the command speed are compared and the error is reduced by using PI controllers. The basic formula used for this is as in Eq. 11, and the circuit for pi controller is shown in fig 3.

$$
\frac{Y(t)}{E(t)}=K_{\mathrm{p}} e(t)+K_{\mathrm{i}} \int e(t) d t
$$

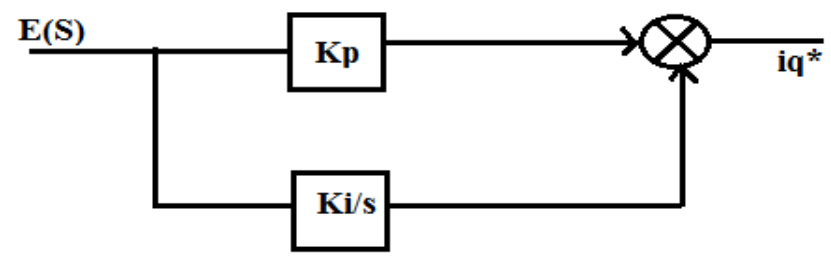

Fig 3 PI controller

Mathematical model of the system is mandatory for these works which limits the use of PI controller. The drawback of tuning PI controller which is very complex is then eliminated by the invention of Fuzzy Logic controller.

\subsection{Fuzzy Logic Controller}

The role of PI controller is replaced by Fuzzy controller which works on rule base. The Fuzzy controller observes the pattern of error signal and correspondingly updates the output so that actual speed reaches the command speed. Based on the input-output relations an IF-THEN rule base is designed for the controller. The basic FLC contains Fuzzification, inference engine and defuzzification. The error and change in error of speed are given as inputs to the controller and are converted into fuzzy variables. On performing rule base, the output is obtained and then is converted to the normal variables which involve defuzzification. The process of implementing fuzzy controller is shown in fig 4 . The rule base of the controller is tabulated in table 1 .

Table 1: IF-THEN rule base

\begin{tabular}{|l|l|l|l|l|l|l|l|}
\hline E & NB & NM & NS & Z & PS & PM & PB \\
\cline { 1 - 5 } CE & & & & & & & \\
\hline NB & NVB & NVB & NB & NM & NS & Z & PS \\
\hline NM & NVB & NB & NB & NM & NS & Z & PS \\
\hline NS & NB & NB & NM & NS & Z & PS & PM \\
\hline Z & NB & NM & NS & Z & PS & PM & PB \\
\hline PS & NM & NS & Z & PS & PM & PB & PB \\
\hline PM & NS & Z & PS & PM & PB & PB & PVB \\
\hline PB & Z & PS & PM & PB & PB & PVB & PVB \\
\hline
\end{tabular}


Therefore PI controller and the FLC controller are the conventional controllers used for the speed control of induction motor drives. Recently, ANN and GA techniques were developed for the control.

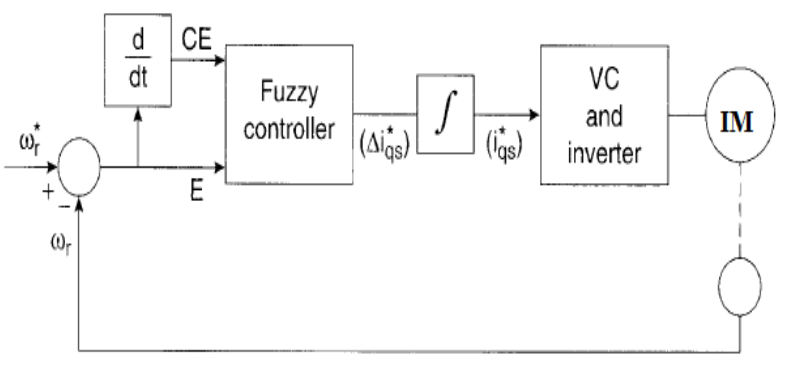

Fig 4 Basic diagram of Fuzzy controller

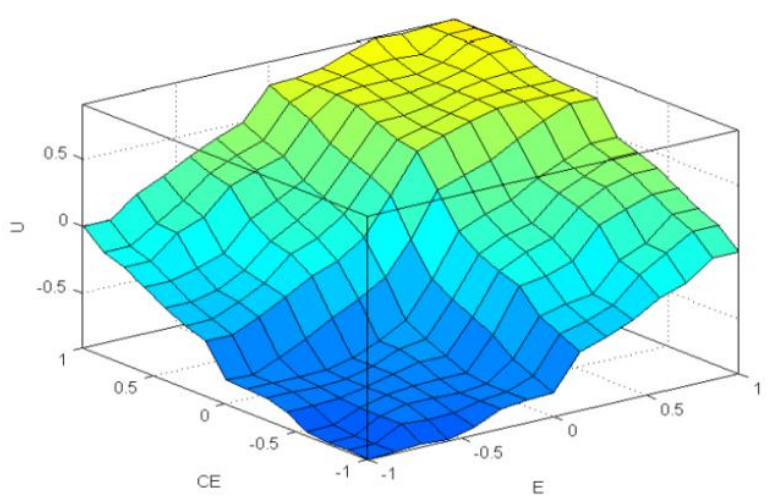

Fig 5 Surface view of rule base

In this paper ANN based control is proposed for the better performance of induction motor drive. The drawback of Fuzzy Control is that it is less suitable for the complex controlled systems to form rules. If the reliable data is not available, then the fuzzy logic implementation will be quite difficult . Further, some adaptive techniques or learning techniques drawn from AI are developed using neural networks to overcome the drawbacks of Fuzzy Controller.

\section{ANN BASED CONTROLLER}

Artificial Neural Network is the general form AI for emulating the human thinking process compared to the FLC. Here, in this paper the speed error is given as input to it. The network consists of input nodes, two hidden layers and one output layer. There is no strict method to find how many neurons should be there in hidden layer. Numbers of neurons in hidden layer are obtained in trial and error method. The weights in network are adjusted during the training procedure. Two layer feed forward neural network is used in this paper. In the two hidden layers one has Tansigmoid function and the other has pure linear transfer function. The basic diagram that explains $\mathrm{NN}$ is as in Fig 6 . With a set of input data, the network can be trained to obtain desired output pattern.

This network has the capability to learn just like human brain because of the intelligence contributed by the weights.

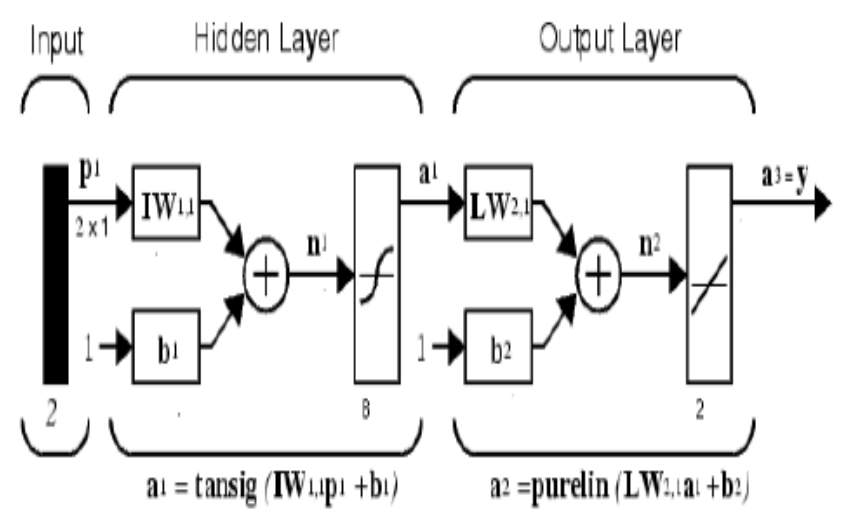

Fig 6 Neural Network model

The two speed inputs are applied to the first hidden layer which passes through tan sigmoid function. Its output is then fed to second hidden layer for getting the required output. In $\mathrm{NN}$, the output of the layer is calculated using the Eq.12.

$$
z=\sum_{i=1}^{n} w_{i} x_{i}-b
$$

Where $w_{i}$ denotes the weights, $x_{\mathrm{i}}$ denotes the inputs and $b$ denotes the bias for the neuron. The processing done by the neural network with activation function is as follows in Eq.13

$$
y=f(z)=f\left(\sum_{i=1}^{n} w_{i} x_{i}-b\right)
$$

With its unique property of performing overall function in case of failure of a single neuron functioning, it proved best performance.

So, they are very robust to the error or failure. In this paper ANN is applied to control the currents of induction motor drive successfully and observed better results than the other conventional controllers.

\section{PROPOSED IVCIM MODEL}

The proposed indirect vector control of induction motor with conventional PI controller, Fuzzy controller is modelled using the Matlab/simulink. The results are compared with the ANN based controller implementation. The developed simulink model is shown in Fig.7.

In the speed control block, the PI controller is first developed and the results are observed. Then it is replaced with fuzzy controller and then with ANN controller. 


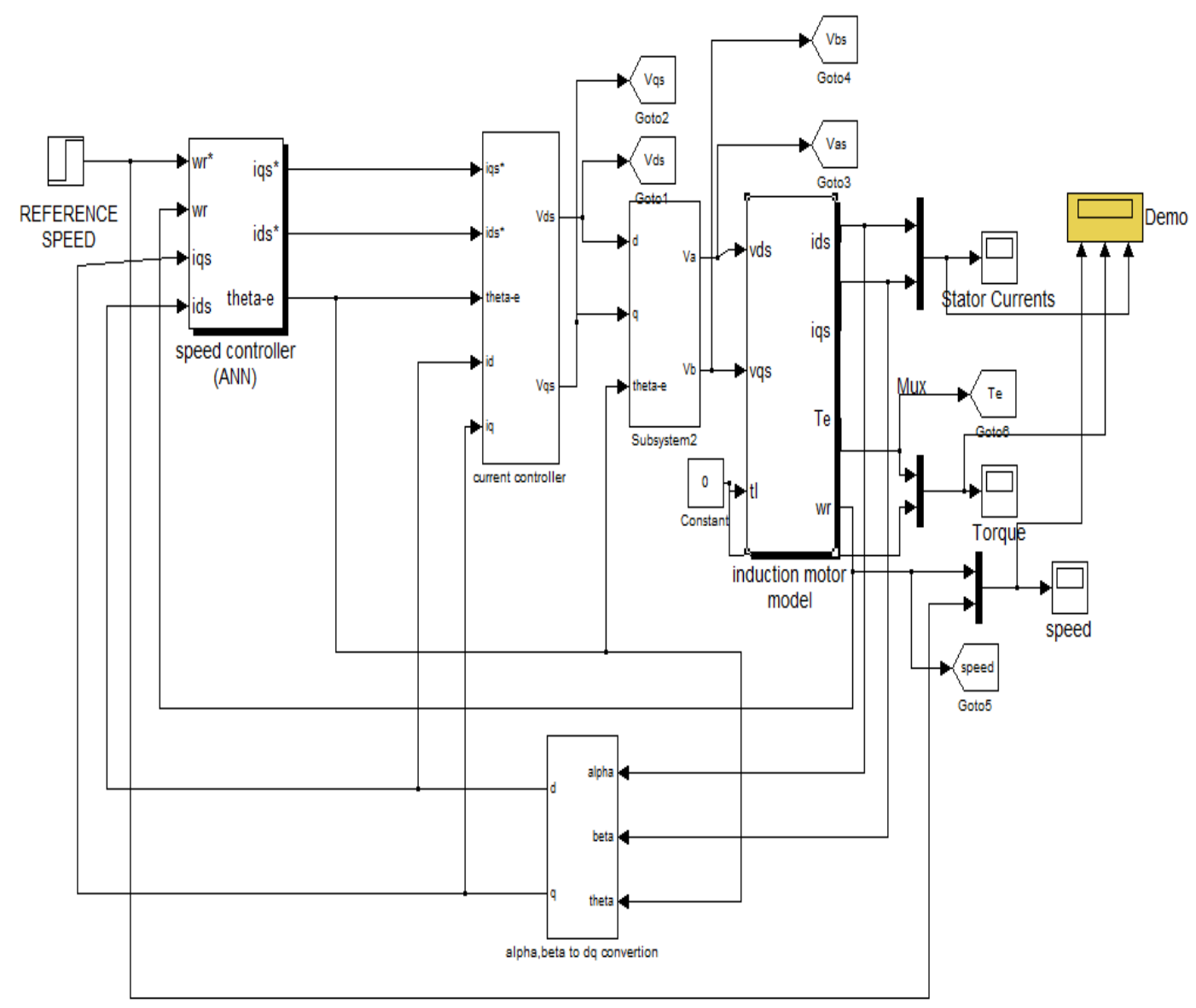

Fig 7 IVCIM with ANN

\subsection{Results}

The induction motor output results with PI controller, Fuzzy controller and ANN controller are obtained using simulation and are analysed. The results are shown in below figures.

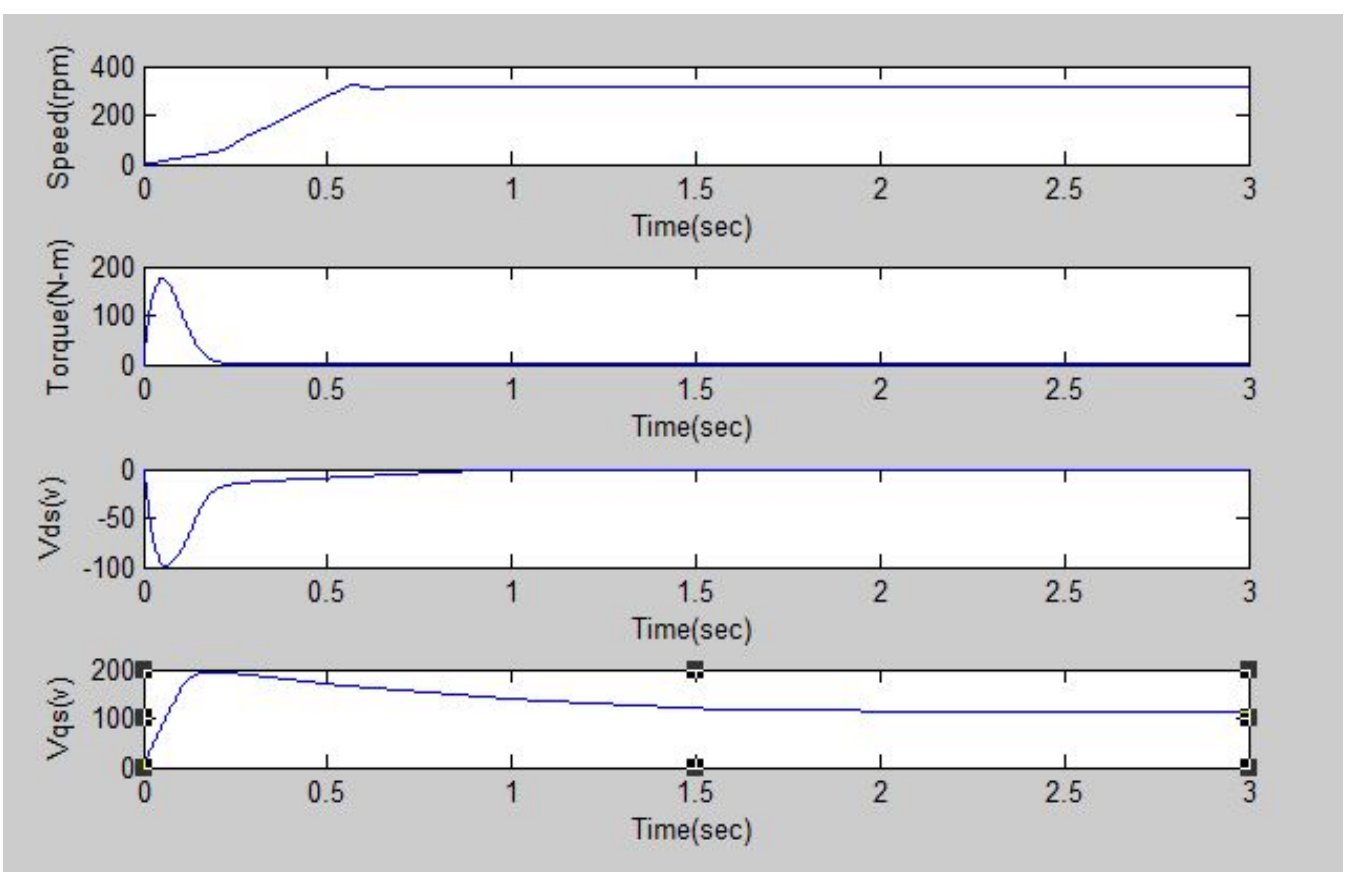

Fig 8 Outcomes of drive with PI controller 
It is observed from the results that, the pi controller tunes the actual speed with reference speed at $0.9 \mathrm{sec}$ with some overshoot.

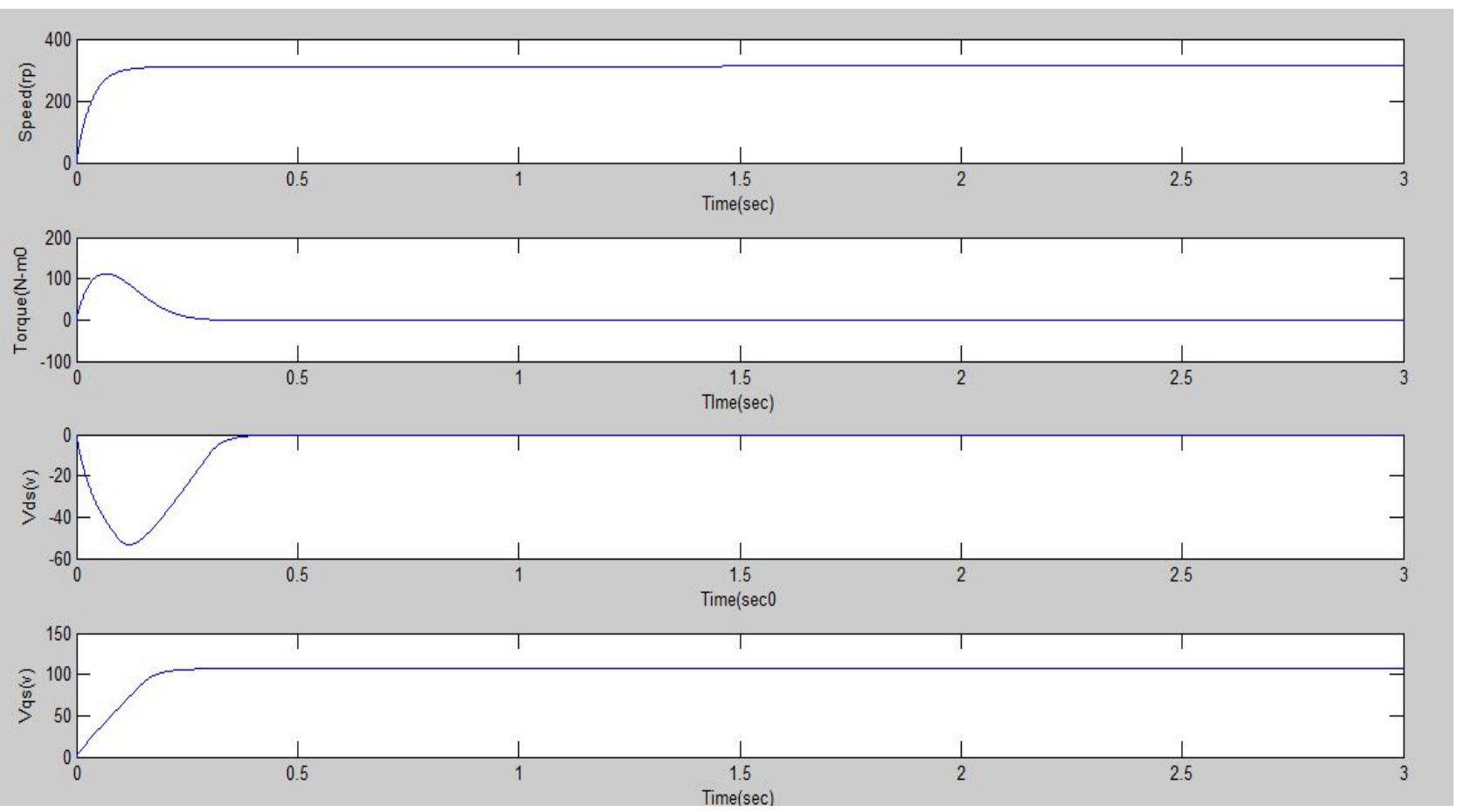

Fig 9 Outcomes of motor drive with fuzzy controller

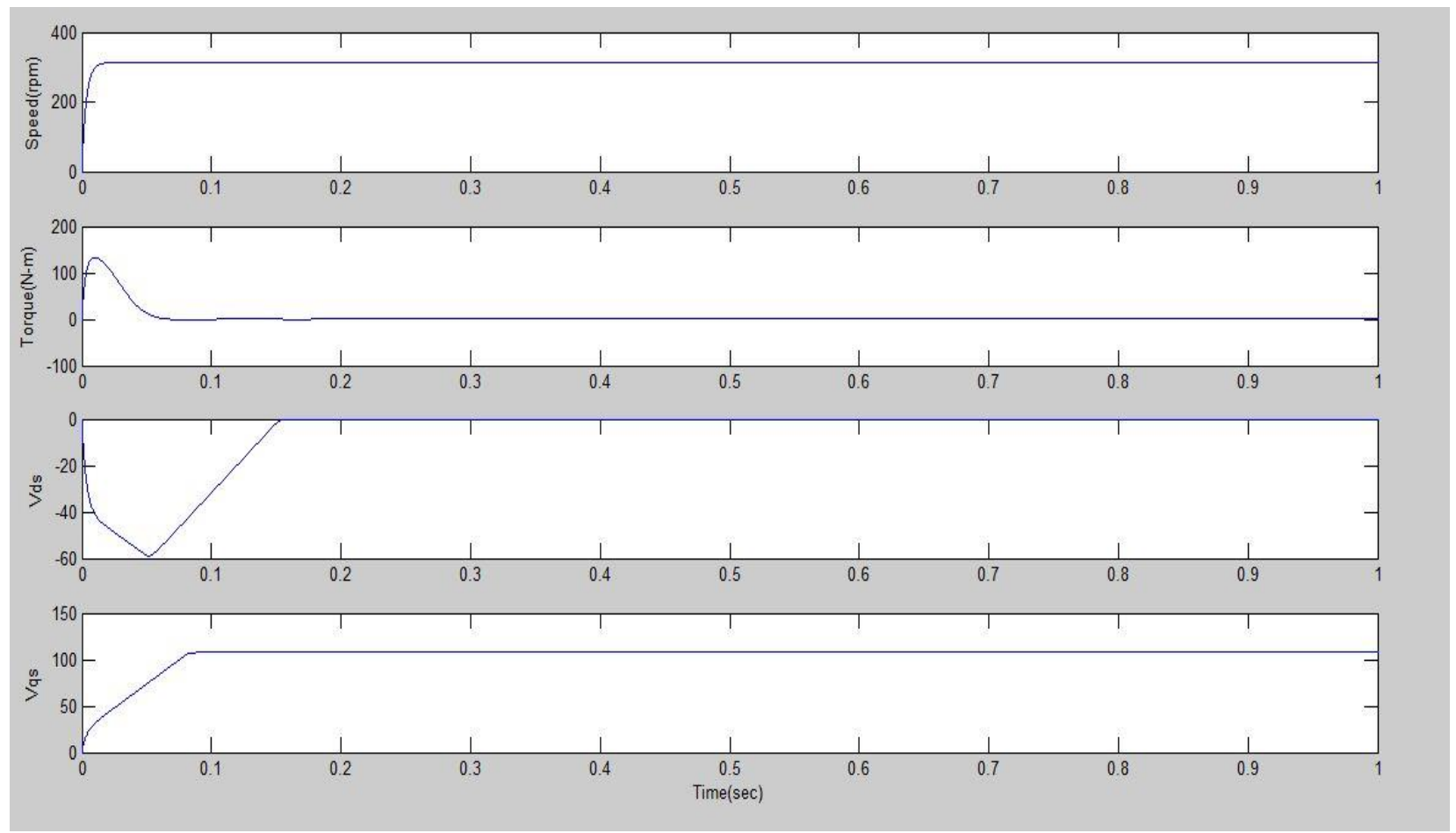

Fig 10 Results obtained with ANN controller

Table 2 Summary of Results

\begin{tabular}{|l|l|l|}
\hline Controller & $\begin{array}{l}\text { Rise Time } \\
\text { (sec) }\end{array}$ & $\begin{array}{l}\text { Settling } \\
\text { Time (sec) }\end{array}$ \\
\hline PI & 0.9 & 0.8 \\
\hline Fuzzy PI & 0.1 & 0.3 \\
\hline ANN & 0.03 & 0.04 \\
\hline
\end{tabular}




\section{CONCLUSION}

An Artificial intelligence based approach was used in this paper to control the speed of the induction motor drive. Between the conventional PI controller and the Fuzzy Logic controller, FLC proved to be better than PI. Response with PI controller has overshoot and takes more settling time to reach rated value. But with the implementation of ANN, the simulation results gave further more improved performance.

\section{REFERENCES}

[1] S Kumar, Prathibha Tiwari, “ Indirect vector control of induction motor using ANN with PI controller, IJIREEICE, Vol.3, 2015

[2] Rani S, Victore George," Performance of ANN based Induction motor drive", IJACTE, Vol.2, pp. 23192526, 2013

[3] Ashutosh Mishra, Prashanth Choudhary, "Speed control of an induction motor by using indirect vector control method", IJETAE, Vol.2, Issue 12, 2012, pp. 144-150.

[4] Vinod Kumar, R. R. Joshi, "Hybrid Controller based Intelligent SpeedControl of Induction Motor", Journal of Theoretical and Applied Information Technology, December 2006, Vol. 3. 